\title{
Fetal growth restriction and the risk of perinatal mortality-case studies from the multicentre PORTO study
}

Julia Unterscheider ${ }^{1 *}$, Keelin O'Donoghue ${ }^{2}$, Sean Daly ${ }^{3}$, Michael P Geary ${ }^{4}$, Mairead M Kennelly, Fionnuala M McAuliffe ${ }^{6}$, Alyson Hunter ${ }^{7}$, John J Morrison ${ }^{8}$, Gerard Burke ${ }^{9}$, Patrick Dicker ${ }^{10}$, Elizabeth C Tully ${ }^{1}$ and Fergal D Malone ${ }^{1}$

\begin{abstract}
Background: Intrauterine growth restriction (IUGR) is the single largest contributing factor to perinatal mortality in non-anomalous fetuses. Advances in antenatal and neonatal critical care have resulted in a reduction in neonatal deaths over the past decades, while stillbirth rates have remained unchanged. Antenatal detection rates of fetal growth failure are low, and these pregnancies carry a high risk of perinatal death.

Methods: The Prospective Observational Trial to Optimize Paediatric Health in IUGR (PORTO) Study recruited 1,200 ultrasound-dated singleton IUGR pregnancies, defined as EFW $<10^{\text {th }}$ centile, between $24^{+0}$ and $36^{+6}$ weeks gestation. All recruited fetuses underwent serial sonographic assessment of fetal weight and multi-vessel Doppler studies until birth. Perinatal outcomes were recorded for all pregnancies. Case records of the perinatal deaths from this prospectively recruited IUGR cohort were reviewed, their pregnancy details and outcome were analysed descriptively and compared to the entire cohort.

Results: Of 1,116 non-anomalous singleton infants with EFW $<10^{\text {th }}$ centile, 6 resulted in perinatal deaths including 3 stillbirths and 3 early neonatal deaths. Perinatal deaths occurred between $24^{+6}$ and $35^{+0}$ weeks gestation corresponding to birthweights ranging from 460 to 2260 grams. Perinatal deaths occurred more commonly in pregnancies with severe growth restriction (EFW $<3^{\text {rd }}$ centile) and associated abnormal Doppler findings resulting in earlier gestational ages at delivery and lower birthweights. All of the described pregnancies were complicated by either significant maternal comorbidities, e.g. hypertension, systemic lupus erythematosus (SLE) or diabetes, or poor obstetric histories, e.g. prior perinatal death, mid-trimester or recurrent pregnancy loss. Five of the 6 mortalities occurred in women of non-Irish ethnic backgrounds. All perinatal deaths showed abnormalities on placental histopathological evaluation.

Conclusions: The PNMR in this cohort of prenatally identified IUGR cases was 5.4/1,000 and compares favourably to the overall national rate of 4.1/1,000 births, which can be attributed to increased surveillance and timely delivery. Despite antenatal recognition of IUGR and associated maternal risk factors, not all perinatal deaths can be prevented.
\end{abstract}

Keywords: Perinatal mortality, Antepartum stillbirth, Neonatal death, Intrauterine growth restriction, PORTO Study

\footnotetext{
* Correspondence: juliaunterscheider@rcsi.ie

'Obstetrics \& Gynaecology, Royal College of Surgeons in Ireland, Dublin, Ireland

Full list of author information is available at the end of the article
}

\section{() Biomed Central}

(C) 2014 Unterscheider et al.; licensee BioMed Central Ltd. This is an Open Access article distributed under the terms of the Creative Commons Attribution License (http://creativecommons.org/licenses/by/2.0), which permits unrestricted use, distribution, and reproduction in any medium, provided the original work is properly credited. The Creative Commons Public Domain Dedication waiver (http://creativecommons.org/publicdomain/zero/1.0/) applies to the data made available in this article, unless otherwise stated. 


\section{Background}

Suboptimal intrauterine growth affects up to $10 \%$ of pregnancies and confers an increased risk of perinatal morbidity and mortality. The perinatal outcome of IUGR fetuses is largely dependent on the severity of growth restriction with those below the $3^{\text {rd }}$ centile and/ or abnormal umbilical artery Doppler measurements at greatest risk of adverse outcome [1]. Other important prenatal determinants of perinatal outcome are gestational age at delivery and birthweight, with best prospects of morbidity-free survival to hospital discharge at weights over 800 grams and gestational ages over 29 weeks [2].

Advances in obstetrical and critical neonatal care are reflected in a substantial decrease in the overall perinatal mortality rate (PNMR) in high income countries. While this effect is mainly seen in a reduction of early neonatal deaths, stillbirth rates have remained largely unchanged over the past years [3,4]. In addition to congenital abnormalities, more recent reports have identified fetal growth restriction as one of the main contributors to perinatal mortality [5].

Ireland consistently reports highest birth rates among European countries with 16.8 births per 1,000 population (compared to $13.0 / 1,000$ in the UK and 8.3/1,000 in Germany) [6]. In 2011, there were 74,265 births and 456 perinatal deaths in Ireland [7]. Stillbirths and early neonatal deaths accounted for $70 \%(n=318)$ and $30 \%(n=138)$ of perinatal deaths respectively, corresponding to a PNMR of 6.1/1,000 births. Of the 456 perinatal deaths, 155 (34\%) were attributed to congenital structural or genetic abnormalities (corrected PNMR 4.1/1,000). Over 50\% of infants affected by perinatal deaths in 2011 were identified as having birthweights below the $10^{\text {th }}$ customised centile and only $30 \%$ of those were suspected antenatally [7].

Current antenatal detection rates of IUGR are reported at 25 to $36 \%[8,9]$. Therefore, a preventative strategy to reduce stillbirths is to improve the antenatal detection of fetal growth failure. The risk of stillbirth in pregnancies with prenatally identified IUGR is $1 \%$ (9.7/ 1,000 births). Pregnancies with unrecognized IUGR carry an over 8-fold increased risk of stillbirth (SB) when compared to pregnancies without IUGR (19.8 versus 2.4/1,000 births) [5]. Whenever IUGR is diagnosed prenatally, increased surveillance and timely delivery aims to improve perinatal outcome in IUGR, balancing the risk of antepartum stillbirth by remaining in utero and iatrogenic prematurity potentially causing significant morbidity or neonatal death by too early intervention.

This paper describes the 6 perinatal mortality cases which occurred in the setting of a multicentre prospective study of 1,200 pregnancies with prenatally identified IUGR defined as EFW below the $10^{\text {th }}$ centile $[1,10]$.

\section{Methods}

The Prospective Observational Trial to Optimise Paediatric Health in IUGR (PORTO) is a national prospective study conducted at seven largest maternity units in Ireland. Intrauterine growth restriction (IUGR) was defined as EFW below the $10^{\text {th }}$ centile based on sonographic measurements of the fetal head circumference $(\mathrm{HC})$, bi-parietal diameter (BPD), abdominal circumference (AC) and femur length (FL) according to population-based growth standards (Hadlock-4) [11]. Between January 2010 and June 2012, the PORTO Study recruited 1,200 ultrasound-dated singleton pregnancies between $24^{+0}$ and $36^{+6}$ weeks' and an EFW equal or greater than 500 grams. Fetuses with congenital structural or chromosomal abnormalities were excluded from the final analysis. Ethical approval was obtained at each participating site, and a written informed consent was given by all study participants.

Women with IUGR pregnancies underwent serial sonographic evaluation of fetal weight at 2-weekly intervals until birth including evaluation of amniotic fluid volume and multi-vessel Doppler assessment. Details on Doppler assessment and reference ranges are described in a previous publication [10]. In cases of absent (AEDF) or reversed end-diastolic (REDF) in the UA, the patient was admitted to hospital and daily electronic fetal heart rate (FHR) monitoring with computerised analysis of short term variation according to Dawes/Redman criteria (Sonicaid Team Fetal Monitor with FHR analysis, MDI Medicare, Ireland) was carried out. Corticosteroids to promote fetal lung maturation and reduce perinatal morbidity were administered as a single course between 24 and 36 weeks' gestation if delivery was likely within one week. Given that the PORTO Study was observational and descriptive in nature, there were no pre-specified management or delivery criteria and all decisions were made by the lead clinician managing each case reflecting real world clinical practice. All study participants received the same surveillance (2-weekly sonogram or more frequently as deemed necessary) with the aim to describe differences in outcome. There was however general agreement among clinicians to deliver AEDF cases no later than 34 weeks' gestation.

The perinatal mortality rate (PNMR) was calculated by the number of stillbirths and neonatal deaths per 1,000 live births and stillbirths (all births were $\geq 24$ weeks gestation and weighed $\geq 500$ grams). The PNMR was corrected for congenital abnormalities.

All cases were prospectively recruited and medical records of all mortalities were reviewed retrospectively for this descriptive analysis. Comparisons were made to the entire cohort, and demographic and pregnancy course and outcome data were recorded along with a review of placental histopathology.

Placental abnormalities were categorised according to Redline criteria [12] into villous developmental 
abnormalities (distal villous dysmaturity/immaturity, abnormal placental shape/position, chorangiosis), maternal vascular pathology, fetal vascular pathology and inflammatory lesions (acute chorioamnionitis, chronic villitis of unknown aetiology). Maternal vascular injuries include infarction, retroplacental haemorrhage and increased perivillous fibrinoid deposition (maternal floor infarction). Fetal vascular injuries include true cord knots, cord hypercoiling, abnormal cord insertion, single umbilical artery and fetal thrombotic vasculopathy.

Due to the small sample size, formal statistical comparisons and modelling (such as logistic regression) are underpowered. Therefore, statistically non-significant findings have the potential to be misleading. Furthermore, due to the biases associated with retrospective selection of controls and the potential for overmatching with a small case-set, the mortalities are presented as a case-series rather than as a case-control study. Consequently, the cases have been described individually (as shown in Figure 1), where each pregnancy/mortality case has its own context.

\section{Results}

There were 6 mortalities including 3 stillbirths (SB) and 3 early neonatal deaths (NND) within the entire cohort of 1,116 non-anomalous singleton pregnancies completing the study protocol. This corresponds to a PNMR of $5.4 / 1,000$. The maternal demographics, baseline characteristics and sonographic findings are described in Table 1. Not surprisingly, perinatal deaths occurred more commonly in pregnancies with severe growth restriction $\left(\mathrm{EFW}<3^{\text {rd }}\right.$ centile) and associated abnormal Doppler findings resulting in earlier gestational ages at delivery and lower birthweights.

Stillbirths occurred at gestational ages between $24^{+6}$ and $31^{+3}$ weeks with birthweights ranging from 460 to 740 grams (mean 607 grams). All stillbirths had estimated weights below the $3^{\text {rd }}$ centile and AEDF in the umbilical artery, and they occurred during inpatient care. The median time from study recruitment to diagnosis of intrauterine demise was 12 days (range 0-45 days). One woman had placenta praevia associated with recurrent antepartum haemorrhages and one woman had severe pre-eclampsia on the background of essential hypertension. Two women had previous pregnancies that resulted in perinatal deaths (one NND due to abruption at 36 weeks, one with unexplained SB at term), and all women with stillbirths were non-Irish nationals.

All cases of neonatal deaths were delivered by emergency caesarean section between $27^{+4}$ and $35^{+0}$ weeks

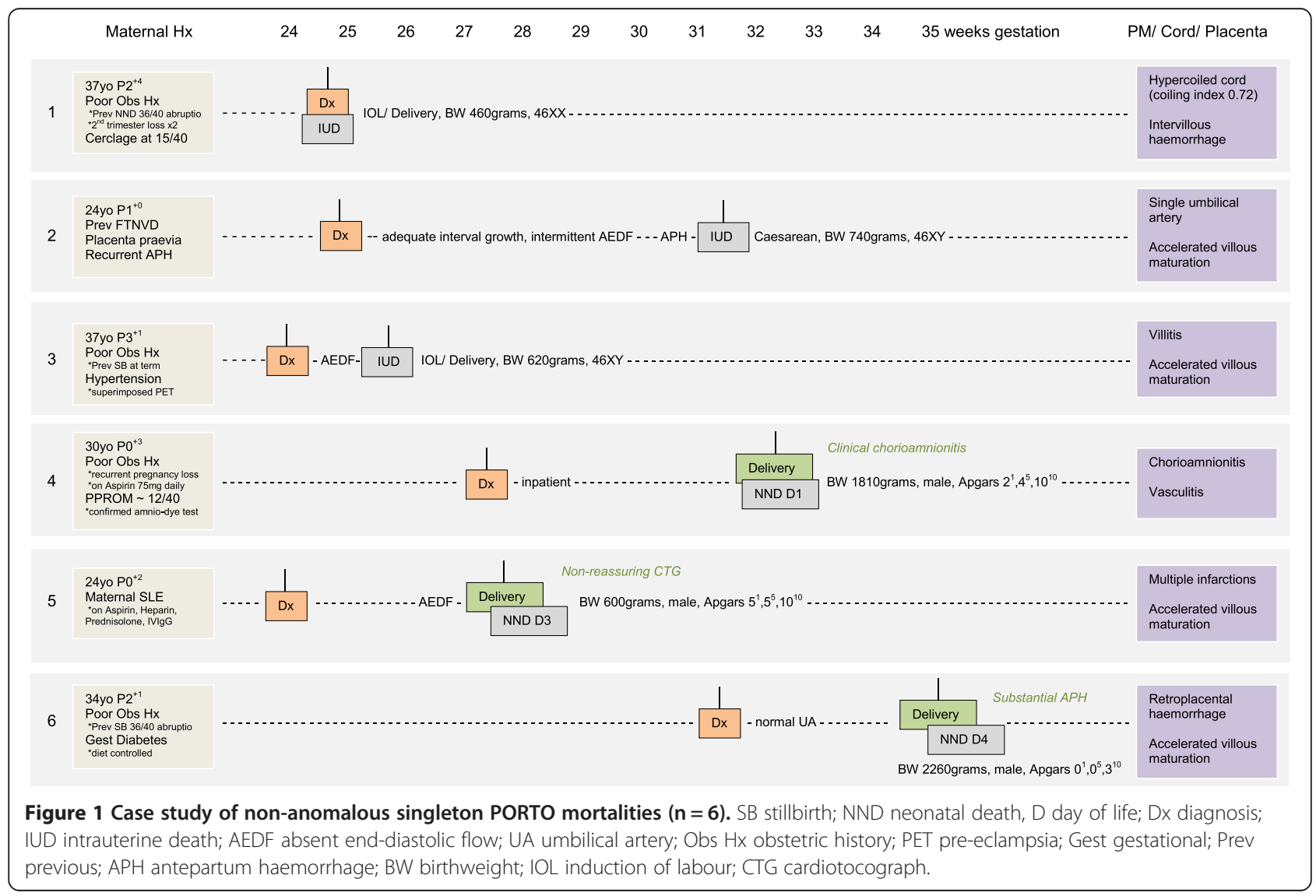


Table 1 Maternal demographics, baseline characteristics and sonographic findings

\begin{tabular}{|c|c|c|}
\hline & $\begin{array}{l}\text { Perinatal } \\
\text { mortalities } \\
(n=6)\end{array}$ & $\begin{array}{l}\text { Alive at hospital } \\
\text { discharge } \\
(n=1,110)\end{array}$ \\
\hline Maternal age & $31.5 \pm 6.3$ & $29.9 \pm 6.0$ \\
\hline Advanced maternal age $>35$ years & $2(33 \%)$ & $225(20 \%)$ \\
\hline Nulliparity & $2(33 \%)$ & $298(45 \%)$ \\
\hline Smoker & 0 & $261(24 \%)$ \\
\hline $\mathrm{BMI}>30 \mathrm{~kg} / \mathrm{m}^{2}$ & $3(50 \%)$ & $122(11 \%)$ \\
\hline Hypertension/Pre-eclampsia & $1(17 \%)$ & $148(13 \%)$ \\
\hline Placental abruption & $2(33 \%)$ & $14(1 \%)$ \\
\hline Assisted reproductive techniques & 0 & $16(1 \%)$ \\
\hline Maternal diabetes & $1(17 \%)$ & $17(2 \%)$ \\
\hline GA at enrolment & $26.1 \pm 2.7$ & $30.1 \pm 3.9$ \\
\hline GA at delivery & $29.4 \pm 3.8$ & $37.8 \pm 2.9$ \\
\hline Last scan EFW $<3^{\text {rd }}$ centile & $5(83 \%)$ & $421(38 \%)$ \\
\hline Last scan EFW $<10^{\text {th }}$ centile & $6(100 \%)$ & $834(75 \%)$ \\
\hline $\begin{array}{l}\text { Last scan EFW }<10^{\text {th }} \text { customised } \\
\text { centile }\end{array}$ & $6(100 \%)$ & $794(72 \%)$ \\
\hline Birthweight $<3^{\text {rd }}$ centile & $4(67 \%)$ & $507(46 \%)$ \\
\hline $\begin{array}{l}\text { Abnormal UA Doppler } \\
\left(\mathrm{PI}>95^{\text {th }}, \text { AEDF) }\right.\end{array}$ & $4(67 \%)$ & $508(46 \%)$ \\
\hline AEDF in the UA & $4(68 \%)$ & $72(6 \%)$ \\
\hline
\end{tabular}

$P I$, pulsatility index; $U A$, umbilical artery; $A E D F$, absent end-diastolic flow; $E F W$ estimated fetal weight; $G A$, gestational age.

gestation corresponding to birthweights between 600 and 2260 grams (mean 1147 grams). The median time of antenatal surveillance was 25 days (range 24-34 days). As outlined in Figure 1, delivery \#4 was for clinical chorioamnionitis due to prolonged preterm rupture of membranes from 12 weeks gestation. The neonate died on day 1 of life due to pulmonary hypoplasia. A prolonged bradycardia on antenatal fetal heart rate monitoring prompted delivery of a severely growth restricted infant $<3^{\text {rd }}$ centile and umbilical artery AEDF in case \#5. The mother received inpatient care for active SLE and the neonate died on day 3 of life. Neonatal death \#6 resulted from a recurrent placental abruption at 35 weeks gestation one week prior to her planned delivery; in this case neonatal intensive care was withdrawn after 4 days due to grade 3 hypoxic ischemic encephalopathy (HIE) and multi-organ failure.

Figure 1 summarises the obstetric history and maternal risk factors and outlines the pregnancy course in detail, including information on delivery indication and outcome. The table also shows information derived from post-mortem examination, cord and placental histopathological evaluation. All of the described pregnancies were complicated by either significant maternal comorbidities, in particular hypertension, SLE or diabetes, or poor obstetric histories. Maternal histories were significant for previous IUGR, recurrent or late pregnancy loss, NND or SB. Two thirds of patients $(n=4)$ had a poor obstetric history including 3 women with term mortalities in prior pregnancies (2 placental abruptions and 1 unexplained stillbirth at term) and 1 woman with a history of recurrent first trimester miscarriages. Four of the 6 mortalities occurred in women of non-Irish ethnic backgrounds. All cases showed placental or cord pathologies including maternal and fetal vascular injuries, placental developmental abnormalities or inflammatory lesions.

The majority of perinatal deaths $(n=5)$ were associated with an EFW $<3^{\text {rd }}$ centile and abnormal UA Doppler velocimetries in the umbilical artery. The 2 perinatal deaths with normal UA Doppler were attributed to pulmonary hypoplasia and histologically confirmed chorioamnionitis due to prolonged preterm rupture of the membranes which and to a recurrent placental abruption at 35 weeks gestation.

\section{Discussion}

The striking and common feature of all perinatal death cases in our study is that they more commonly occurred in non-Irish national women attending for antenatal care with poor obstetric histories and coexistent maternal comorbidities. These findings are in agreement with other reports which found that ethnic minorities are an overrepresented group among mothers who experience perinatal deaths $[7,13]$. In particular, women with a history of adverse pregnancy outcomes, such as recurrent pregnancy loss, stillbirth or perinatal deaths, are at higher risk of recurrent adverse outcomes with fewer than $25 \%$ pregnancies resulting in surviving infants [14].

Perinatal deaths occurred more commonly among infants with severe growth restriction and associated abnormal umbilical artery Doppler values which highlights the fact that fetuses with EFW $<3^{\text {rd }}$ centile and/or abnormal UA Doppler are a greater risk of poor perinatal outcome therefore making this a more appropriate cut-off for the definition of pathological growth restriction [1].

A detailed evaluation of the cord and placenta is useful in determining the underlying causes which have led to an IUGR diagnosis. Histopathological examination was carried out for all perinatal deaths in our study and interestingly showed abnormal findings in $100 \%$ of cases including maternal and fetal vascular injuries, placental developmental abnormalities or inflammatory lesions. Unfortunately, there are no specific antenatal screening or preventative strategies for cord and placental abnormalities. Together with maternal factors, this information can however be utilised when caring for women in a subsequent pregnancies.

The PNMR in this IUGR cohort compares favourably to the national corrected PNMR of 4.1/1,000. The 
relatively low PNMR in this high risk population of IUGR fetuses can be explained by (i) the intensive prenatal surveillance within a research setting attributed to the Hawthorne effect, which has been described as a confounding factor for performance in clinical observational studies and (ii) the exclusion of pregnancies at most severe spectrum of disease (i.e. EFW $<500$ grams at or over 24 weeks gestation). Despite the antenatal recognition of maternal risk factors and diagnosis of IUGR with initiation of increased surveillance and inpatient management, not all perinatal deaths in our study were prevented. Some of these deaths occurred during inpatient care or were due to unforeseen acute complications such as placental abruption, antepartum haemorrhage in the presence of placenta praevia or acute chorioamnionitis. However the increased surveillance certainly benefited the majority of women and babies enrolled in PORTO reflected in the low PNMR in this high risk cohort which was comparable to the overall national rate.

A limitation of our study concerns the inability to comment on antenatal detection rates of IUGR in Irish maternity units as data on this was not recorded. Prenatal recognition of IUGR remains the main challenge in daily obstetric practice. Current detection rates of IUGR in the antenatal setting are at best $36 \%$ and future research needs to urgently focus on how we can improve these detection rates. Clinical examination, abdominal palpation and fundal height measurement have limited accuracy in identifying IUGR prenatally and serial ultrasound scanning from 26 to 28 weeks of gestation has been proposed in patients with risk factors [15]. A care model whereby every pregnant patient receives an ultrasound scan at least 4 weekly intervals would improve identification of growth failure based on population and customised growth standards. Given that IUGR can also occur in infants born with birthweights above the $10^{\text {th }}$ centile cut-off, this approach would also allow to comment on growth trajectories which have been identified as an important factor in the prediction of morbidity and mortality outcomes [16]. The relevance to clinical practice in reducing perinatal morbidity and mortality could be the subject of future research studies comparing various models of antenatal care. This will impact on resource issues, increase obstetric intervention but no doubt will have an impact on the antenatal detection and reduction in perinatal deaths. At the present time, we propose a management algorithm outlined in Figure 2 [17].

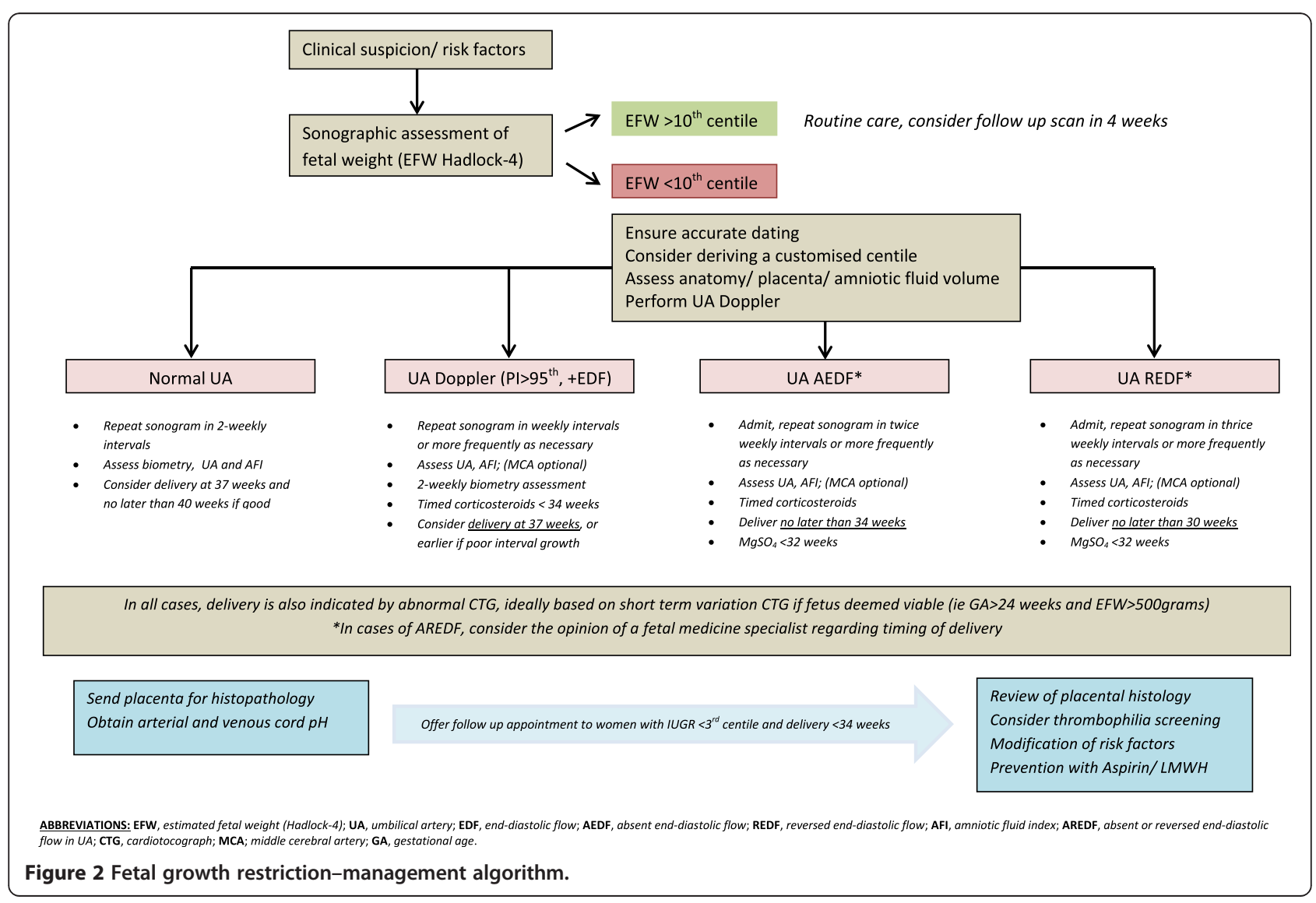




\section{Conclusions}

Fetal growth restriction is the single most important contributor to perinatal mortality in non-anomalous fetuses [5]. Significant research efforts have gone into improving the diagnosis and definition of IUGR, surveillance and antenatal management, however uncertainties regarding the optimal timing of delivery in IUGR persist.

Vigilance towards antenatal risk factors for poor pregnancy outcome is important for the optimal management of IUGR pregnancies. The focus of future research needs to urgently address how we can improve the antenatal detection of IUGR. Prenatal recognition of IUGR dramatically improves pregnancy outcomes allowing for close monitoring and timely delivery. Nevertheless, even if recognised, not all perinatal deaths can be prevented.

\footnotetext{
Abbreviations

IUGR: Intrauterine growth restriction; PORTO: Prospective Observational Trial to Optimize Paediatric Health in IUGR; SB: Stillbirth; IUD: Intrauterine death; UtA: Uterine artery; UA: Umbilical artery; NND: Neonatal death; APH: Antepartum haemorrhage; BW: Birthweight; Dx: Diagnosis; PET: Preeclampsia; PPROM: Prolonged preterm rupture of membranes; AEDF: Absent end-diastolic in the UA; REDF: Reversed end-diastolic flow in the UA; CTG: Computerised short term variation cardiotocograph; HIE: Hypoxic ischaemic encephalopathy; PNMR: Perinatal mortality rate.
}

\section{Competing interests}

The authors declare that they have no competing interests. The study was funded by the Health Research Board (HRB) and Friends of the Rotunda.

\section{Authors' contributions}

All authors have made substantial contributions to conception, design and data acquisition of the PORTO study. KOD had the idea for this particular analysis. JU drafted the manuscript. All co-authors revised it critically for important intellectual content. PD performed the statistical analysis. All authors read and approved the final manuscript.

\section{Authors' information \\ The PORTO Study was conducted by the Perinatal Ireland Research Consortium, a nationwide collaborative research network comprising of the seven largest academic obstetric centres in Ireland. Those include the Rotunda Hospital Dublin, the Coombe Women's and Infants University Hospital Dublin, the National Maternity Hospital Dublin, the Cork University Maternity Hospital, the Royal Jubilee Maternity Hospital Belfast, the Galway University Hospital and the Mid-Western Regional Maternity Hospital Limerick. FDM is the Chair of Perinatal Ireland. $J U$ is the lead researcher of the PORTO study and clinical PhD student under the supervision of KOD and FDM.}

\section{Acknowledgements}

The authors thank Fiona Manning, original Perinatal Ireland research manager, and the team of Perinatal Ireland research sonographers Fiona Cody, Hilda O'Keefe, Emma Doolin, Cecelia Mulcahy, Azy Khalid, Phyl Gargan, Annette Burke, Edel Varden, Wendy Ooi and Amanda Ali.

\section{Author details}

${ }^{1}$ Obstetrics \& Gynaecology, Royal College of Surgeons in Ireland, Dublin, Ireland. 'Obstetrics \& Gynaecology, University College Cork, Cork University Maternity Hospital, Cork, Ireland. ${ }^{3}$ Obstetrics \& Gynaecology, Coombe Women and Infants University Hospital, Dublin, Ireland. ${ }^{4}$ Obstetrics \& Gynaecology, Rotunda Hospital, Dublin, Ireland. ${ }^{5}$ UCD Centre for Human Reproduction, Coombe Women and Infants University Hospital, Dublin, Ireland. ${ }^{6}$ Obstetrics \& Gynaecology, UCD School of Medicine and Medical Science, National Maternity Hospital, Dublin, Ireland. ${ }^{7}$ Obstetrics \& Gynaecology, Royal Jubilee Maternity Hospital, Belfast, Ireland. ${ }^{8}$ Obstetrics \& Gynaecology, National University of Ireland, Galway, Ireland. ${ }^{9}$ Obstetrics \&
Gynaecology, Graduate Entry Medical School, University of Limerick, Limerick, Ireland. ${ }^{10}$ Epidemiology \& Public Health, Royal College of Surgeons in Ireland, Dublin, Ireland.

Received: 11 November 2013 Accepted: 7 February 2014

Published: 11 February 2014

\section{References}

1. Unterscheider J, Daly S, Geary MP, McAuliffe FM, Kennelly MM, Morrison JJ, O'Donoghue K, Hunter A, Burke G, Dicker P, Tully E, Malone FD: Optimizing the definition of intrauterine growth restriction-results of the multicenter prospective PORTO study. AJOG 2013, 208(4):290.e1-6.

2. Baschat AA, Cosmi E, Bilardo CM, et al: Predictors of neonatal outcome in early onset placental dysfunction. Obstet Gynecol 2007, 109:253-261.

3. Lawn JE, Blencowe H, Pattinson R, Cousens S, Kumar R, Ibiebele I, Gardosi J, Day L, Stanton C: Stillbirths: where? When? Why? How to make the data count? Lancet 2011, 377(9775):1448-1463.

4. Cousens S, Blencowe H, Stanton C, Chou D, Ahmed S, Steinhardt L, Creanga AA, Tunçalp O, Balsara ZP, Gupta S, Say L, Lawn JE: National, regional and worldwide estimates of stillbirth rates in 2009 with trends since 1995: a systematic analysis. Lancet 2011, 377(9774):1319-1330.

5. Gardosi J, Madurasinghe V, Williams M, Malik A, Francis A: Maternal and fetal risk factors for stillbirth: population based study. BMJ 2013, 346:f108.

6. National Perinatal Reporting System (NPRS): Health Research and Information Division, the Economic and Social Research Institute. Ireland: Perinatal Statistics Report; 2010.

7. Manning E, Corcoran P, Meaney S, Greene RA, on behalf of the Perinatal Mortality Group: Perinatal Mortality in Ireland Annual Report 2011. National Perinatal Epidemiology Centre: Cork; 2013.

8. McCowan LM, Roberts CT, Dekker GA, et al: Risk factors for small-for gestational-age infants by customised birthweight centiles: data from an international prospective cohort study. BJOG 2010, 117(13):1599-1607.

9. Chauhan SP, Beydoun H, Chang E, et al: Prenatal detection of fetal growth restriction in newborns classified as small for gestational age: correlates and risk of neonatal morbidity. Am J Perinatol 2013. Apr 16. [Epub ahead of print]

10. Unterscheider J, Daly S, Geary MP, McAuliffe FM, Kennelly MM, Morrison J, O'Donoghue K, Hunter A, Burke G, Dicker P, Tully E, Malone FD: Predictable progressive doppler deterioration in IUGR-does it really exist? AJOG 2013. doi:10.1016/j.ajog.2013.08.039.

11. Hadlock FP, Harrist RB, Sharman RS, Deter RL, Park SK: Estimation of fetal weight with the use of head, body, and femur measurements-a prospective study. Am J Obstet Gynecol 1985, 151(3):333-337.

12. Redline RW: Placental pathology: a systematic approach with clinical correlations. Placenta 2008, 29(Suppl A):S86-S91.

13. de Graaf JP, Steegers EA, Bonsel GJ: Inequalities in perinatal and maternal health. Curr Opin Obstet Gynecol 2013 Apr, 25(2):98-108.

14. Friars AE, Luikenaar RA, Sullivan AE, Lee RM, Porter TF, Branch DW, Silver RM: Poor obstetric outcome in subsequent pregnancies in women with prior fetal death. Obstet Gynecol 2004, 104(3):521-526.

15. Royal College of Obstetricians and Gynaecologists (RCOG): Green-top guideline No 31: The investigation and management of the small-forgestational-age fetus. 2nd edition. London: RCOG Press; 2013.

16. Barker ED, McAuliffe FM, Alderdice F, Unterscheider J, Daly S, Geary MP, Kennelly MM, O'Donoghue K, Hunter A, Morrison JJ, Burke G, Dicker P, Tully $E$, Malone FD: The role of growth trajectories in classifying fetal growth restriction. Obstet Gynecol 2013, 122(2, PART1):248-254.

17. Institute of Obstetricians and Gynaecologists, Royal College of Physicians of Ireland and Health Service Executive: Clinical Practice Guideline No 29: Fetal Growth Restriction - Recognition, Diagnosis and Management. Dublin: RCPI; 2014. In press. www.hse.ie.

\section{doi:10.1186/1471-2393-14-63}

Cite this article as: Unterscheider et al:: Fetal growth restriction and the risk of perinatal mortality-case studies from the multicentre PORTO study. BMC Pregnancy and Childbirth 2014 14:63. 